\title{
LA GUERRE SUR LE WEB. LES CADRAGES JOURNALISTIQUES DU CONFLIT LIBYEN DANS LES SITES FRANÇAIS D'ACTUALITÉ
}

\author{
Emmanuel Marty et Franck Rebillard ${ }^{1}$
}

Le web constitue désormais l'un des principaux moyens d'expression publique. Plus particulièrement, le secteur contemporain de l'information d'actualité en ligne accueille ainsi à la fois des médias professionnels et des initiatives d'amateurs, sans compter de puissants infomédiaires tels que Google (Rebillard \& Smyrnaios, 2010). De ce point de vue, la sphère publique numérique pourrait être comparée à une arène, un lieu de concurrences et de contradictions, plutôt qu'à un paisible marché des idées (Peters, 2004). L'offre d'actualités surl'internet résulte de telles différences dans les stratégies éditoriales, politiques et industrielles (Mosco, 2009 ; Fenton, 2010). Dans ce contexte, les sites d'information se positionnent au sein d'un continuum opposant la productivité à la créativité, et la réactivité à la réflexivité. En effet, lorsque l'on pose la question du pluralisme de l'information en ligne, on peut observer une ligne de fracture entre ses acteurs : d'un côté se trouvent des sites d'information jouissant d'un important trafic, avec de fortes contraintes commerciales et étroitement dépendants des agences de presse pour leur production; de l'autre des sites d'information avec une fréquentation bien plus faible, mais plus autonomes, créatifs et souvent moins dépendants de contraintes financières et temporelles.

De précédents résultats obtenus dans le cadre du programme de recherche Ipri (Internet, Pluralisme et Redondance de 1'Information) ${ }^{2}$

1 Emmanuel Marty est Chercheur au Laboratoire de recherche i3M à l'Université Nice Sophia Antipolis. Franck Rebillard est Professeur à l'Institut de la Communication et des Médias à l'Université Sorbonne Nouvelle Paris 3.

2 Le programme de recherche Ipri (Internet, pluralisme et redondance de l'information)

Recherches en communication, $\mathrm{n}^{\circ} 40$ (2013). 
ont montré en quoi le pluralisme pouvait coexister sur le web avec de fortes tendances à la redondance dans l'information délivrée (Smyrnaios et al., 2010). Les actualités semblent à la fois variées et très inégalement distribuées : un nombre restreint de sujets d'actualité, considérés comme les plus importants dans l'agenda médiatique, occupent la majorité des contenus produits en ligne.

Deux raisons principales peuvent expliquer cette situation: la première est la nature de l'information numérique qui permet une reproduction à grande échelle des contenus; la seconde concerne les contraintes particulières de l'économie de l'information en ligne, qui détermine la manière de fonctionner des rédactions. En effet, l'énorme quantité d'information produite et reproduite quotidiennement sur la toile par un large spectre d'entités aboutit à une situation d'offre excédentaire. L'information est systématiquement stockée et/ou traitée par des programmes sur les réseaux informatisés, permettant de procéder à moindre coût à une reproduction massive des contenus. Cette offre de contenus informationnels numériques est alors mise à la disposition de millions d'utilisateurs dans le monde, à travers une multiplicité de canaux et d'outils (flux rss, moteurs de recherche, sites de réseaux sociaux, portails personnalisés, agrégateurs, blogs, etc.) qui, dans leur grande majorité, ne produisent pas de contenu original. De plus, comme l'ont montré de récentes recherches, il existe à présent une tendance lourde à la forte productivité dans les rédactions web (Domingo \& Paterson, 2011). Contraints par des délais très courts de publication et par une sorte d'injonction permanente à l'immédiateté, attenante au web, les journalistes ont tendance, sur la toile, à se concentrer sur la réécriture et la publication, en s'appuyant sur le matériau rédactionnel existant, aux dépens du reportage de terrain (Degand, 2011).

A la lumière de ces constats, on peut considérer que l'accroissement de la quantité d'information qui circule en ligne ne se traduit pas nécessairement par une plus grande diversité en termes journalistiques. Nos précédentes recherches, dans le cadre du programme Ipri (cf. supra), étaient fondées sur une approche de l'agenda-setting (Dearing \& Rogers, 1992) destinée à identifier la variété des sujets abordés par différents sites d'actualité. L'étude présentée dans ce texte vise à analyser la

a reçu le soutien de l'Agence Nationale de la Recherche (ANR-09-JCJC-0125-01b). Le programme réunit des chercheurs en information-communication et informatique des laboratoires CIM (Université Paris 3), ELICO (Université de Lyon), LERASS (Université Toulouse 3), CRAPE (Université Rennes 1), I3M (Université de Nice Sophia Antipolis), GRICIS (UQAM Montréal), LIRIS (INSA Lyon). 
diversité de l'information en ligne, en France, d'un autre point de vue. Son but est de compléter les précédents résultats quantitatifs obtenus en se focalisant sur un sujet particulier : le traitement journalistique de la Révolution libyenne en 2011. Plus précisément, il s'agit d'une analyse lexicométrique de la couverture par les sites français d'actualité du soulèvement contre Muammar Khadafi. Une première analyse quantitative de l'agenda des actualités françaises en ligne, menée parmi plus de 200 sites web du 7 au 17 mars 2011, a montré que ce sujet avait alors été très largement traité. Notre objectif ici est de poursuivre notre analyse de la question du pluralisme dans la perspective d'une analyse des cadres mobilisés par les médias en ligne (Entman, 1993). La question est alors de déterminer si la multiplicité des sites favorise ou non une variété dans les cadres journalistiques, à propos d'un sujet tel que le soulèvement libyen, dominant alors l'agenda. En effet, la notion de pluralisme ne renvoie pas uniquement à l'éventail de sujets abordés par les médias, mais également à la manière dont ces sujets sont couverts, y compris du point de vue des opinions et des débats politiques qui leurs sont attenants.

\section{Cadre Théorique}

\section{L'analyse des cadres et la question du pluralisme dans le discours médiatique}

Depuis les années 1980, l'analyse des cadres médiatiques a produit une importante littérature universitaire aux Etats-Unis (beaucoup moins en Europe et particulièrement en France). L'un des premiers auteurs à avoir utilisé le concept de cadre pour désigner l'activité de construction de sens par les médias est Gitlin (1980, p. 8) : « media frames, largely unspoken and unacknowledged, organize the world both for journalists who report it and, for some important degree, for us who rely on their reports ». L'auteur insiste sur un point important: les cadres ne sont pas des opinions. Plus larges que cela, ils constituent plutôt des espaces conceptuels qui organisent le discours et orientent la construction du sens. Plutôt que des opinions, les cadres peuvent être conçus comme des « panoplies interprétatives » guidant à la fois les activités de production et de compréhension du discours. Si bien qu'un même cadre peut inclure des opinions ou arguments opposés, intégrant en cela un certain degré de controverse, variable d'un cadre à l'autre (Gamson \& Modigliani, 1989). Selon Gamson et Modigliani, pour ce 
qui est du journalisme, les cadres ont tendance à être influencés par les normes et routines professionnelles, que ce soit dans la sélection des sources, dans la définition de la newsworthiness ${ }^{1}$ ou dans l'usage de modèles rédactionnels pour la construction des nouvelles. Pour ces auteurs, comme pour Gitlin, le processus de construction du sens, s'il peut prendre appui sur les discours médiatiques, s'inscrit dans une dynamique complexe : «Each system interacts with the other: media discourse is part of the process by which individuals construct meaning, and public opinion is part of the process by which journalists and other cultural entrepreneurs develop and crystallize meaning in public discourse » (Gamson \& Modigliani, 1989, p. 2).

Un autre point important mentionné par Gamson (1992) est l'existence d'une compétition de cadrages (" framing contest »), dans laquelle différents groupes d'intérêt rivalisent pour imposer leur propre définition des faits dans les médias. Les moyens d'accès aux médias dont disposent ces groupes déterminent leur présence dans les actualités, et par conséquent leur audience auprès du public. A partir de ces différents éléments, Entman (1993, p. 52) a élaboré une définition de ce qu'est pour lui l'activité de cadrage des médias:

To frame is to select some aspects of a perceived reality and make them more salient in a communicating text, in such a way as to promote a particular problem definition, causal interpretation, moral evaluation, and/or treatment recommendation for the item described.

Pour Entman, le monde social est une sorte de kaléidoscope de réalités potentielles, et les médias ont tendance à favoriser certaines d'entre elles, fatalement au détriment d'autres. Ce phénomène est en partie dû au milieu socio-culturel dont sont issus les journalistes, mais aussi à la nature des relations qu'ils entretiennent avec leurs sources et, une fois de plus, aux normes et routines professionnelles du champ journalistique, pouvant mener à un certain nombre de biais dans la construction des nouvelles. Par conséquent, la principale question à laquelle nous nous proposons de répondre dans cette étude, en nous appuyant sur ces éléments théoriques, est de savoir si les différentes catégories de sites web d'information fournissent un large éventail de

1 C'est-à-dire la valeur d'un fait ou d'un témoignage, en termes d'intérêt du public et de rentabilité économique. 
cadres dans leur couverture d'un sujet aussi important que celui du soulèvement en Libye.

\section{La catégorisation des sites web d'information}

De précédents travaux nous ont permis de cartographier le paysage de l'information en ligne française, en identifiant différentes catégories de sites web offrant de l'information (Smyrnaios et al., op. cit.). Nous distinguions alors six catégories de sites : les sites de presse en ligne (versions internet de médias existants), les webzines (publications collectives exclusivement internet), les blogs (publications individuelles exclusivement internet), les sites participatifs (publications collaboratives exclusivement internet), les portails (composantes informationnelles de plateformes multiservices) et les agrégateurs (regroupements automatisés d'informations d'actualité). Cette première catégorisation était principalement fondée sur une typologie proposée par Rebillard (2006), elle-même inspirée par Deuze (2003). Au vu d'un certain nombre de changements progressivement survenus sur le web ces dernières années, cette catégorisation semble toutefois devoir être mise à jour. Tout d'abord, la frontière entre agrégateurs et portails est aujourd'hui beaucoup moins nette, car les deux types de sites utilisent à présent des procédés automatisés pour recueillir et diffuser l'information. Par conséquent, ces deux types de sites ont été fusionnés à l'intérieur d'une nouvelle catégorie, celle des infomédiaires, que l'on peut définir comme réalisant un mélange d'agrégation, de réagencement et de diffusion de contenus produits par des tiers, et qui se positionnent comme des intermédiaires entre la production et la demande d'information (Smyrnaios, 2012). Nous avons également rapproché les webzines et les sites participatifs dans une nouvelle catégorie et sous la dénomination de sites natifs de l'internet. Cette catégorie rassemble tous les sites d'information nés sur l'internet sans être auparavant affiliés à un media traditionnel. Ces sites proposent aujourd'hui pour la plupart, à un degré certes variable, une régulation collective des contenus publiés en lien avec les utilisateurs, rendant caduque la séparation entre webzines et sites participatifs. Par ailleurs, ces sites nés en ligne ont précédemment été désignés sous le vocable de pure players, mais cette dénomination renvoie à des publications exclusivement web alors que, pourtant, certains de ces sites ont par la suite développé des versions papier de leurs publications (exemple : Rue 89). La dénomination de médias natifs de l'internet parait alors plus adéquate pour designer ces 
sites. Nous proposons donc une catégorisation mise à jour et simplifiée distinguant les médias en ligne, les médias natifs de l'internet, les blogs et les infomédiaires. Cela étant, à l'intérieur de chaque catégorie, une attention particulière sera accordée au caractère professionnalisé ou au contraire amateur des publications et de leurs auteurs. Il existe en effet une différence importante entre des blogueurs amateurs tenant leur propre espace de publication et des journalistes professionnels écrivant au sein d'un blog hébergé et détenu par leur journal. De la même façon, au sein des médias natifs de l'internet, le contenu peut être principalement pris en charge par des contributeurs amateurs ou, au contraire, par une rédaction professionnelle.

\section{Méthodologie}

\section{L'échantillon de sites web}

Les sites web choisis sont ceux publiant de l'information générale et politique (relation de faits ou commentaires), et visant une audience d'envergure nationale, en France. Nous avons effectué un recensement de tous les sites correspondant à ces critères. Sur la base d'une liste précédemment constituée entre 2008 et 2010, il a été procédé à une mise à jour manuelle pour les médias en ligne et les infomédiaires, dont le nombre est relativement restreint, ainsi que pour certains médias natifs de l'internet. Cette première opération a abouti à la prise en compte de 14 infomédiaires et de 43 médias en ligne, ce qui constitue un corpus exhaustif en France pour ces catégories des sites d'information d'actualité. Par la suite, à l'aide de plusieurs méthodes de recherche et notamment l'utilisation d'un logiciel de navi-crawling ${ }^{1}$, nous sommes parvenus à identifier 110 blogs et 42 médias natifs de l'internet correspondant à nos critères, amenant le nombre total de sites pris en compte à 209 .

\section{Le corpus d'articles}

Le corpus d'articles analysés ici concerne un sujet d'actualité particulier : le conflit en Libye. Il est alors composé de tous les articles traitant de ce sujet et publiés le mardi 8 mars 2011. Le choix de s'attacher à un sujet et à un jour particuliers est motivé par la volonté d'étudier ce

1 A l'aide du logiciel Navicrawler développé par Web Atlas: http://webatlas.fr/wp/ navicrawler/ 
que Moirand (2007, p.82) a appelé un « moment discursif », c'est-à-dire un enchaînement de " faits qui, soudainement et/ou sporadiquement, donnent lieu à une vaste production discursive dans les médias, parce qu'ils sont de nature à inquiéter, donc à attirer lecteurs et spectateurs, quels qu'ils soient. » Or, une analyse quantitative initiale de l'agenda médiatique en ligne, menée entre le 7 et le 17 mars 2011, a montré que le sujet du conflit libyen constituait l'un des tous premiers sujets traités, en nombre d'articles publiés par les différents sites sur l'ensemble de la période. Nous avons plus précisément concentré notre analyse sur les articles publiés lors de la journée du 8 mars, lors de laquelle la ville de Ras Lanouf fut le théâtre d'une résurgence des violences, rendant plus pressante la question alors posée d'une intervention militaire de l'ONU. Tous les sites n'ont toutefois pas publié sur le sujet ce jour-là, en particulier certains médias natifs de l'internet et certains blogs. $\mathrm{Au}$ final, notre corpus est composé de 179 articles, distribués de la manière suivante sur nos quatre catégories de sites:

\begin{tabular}{|c|c|}
\hline $\begin{array}{c}\text { Catégories } \\
\text { de sites }\end{array}$ & $\begin{array}{c}\text { Nombre d'articles publiés par } \\
\text { catégorie }\end{array}$ \\
\hline Médias en ligne & 123 \\
\hline Infomédiaires & 28 \\
\hline Médias natifs de l'internet & 26 \\
\hline Blogs & 2 \\
\hline Total & $\mathbf{1 7 9}$ \\
\hline
\end{tabular}

Tableau 1. Articles web publiés le 8 mars 2011 sur le conflit en Libye

On remarque immédiatement que certains sites web furent beaucoup plus productifs que d'autres sur le sujet. Par exemple, la catégorie des médias en ligne a publié 123 articles, là où les blogs, pourtant en nombre supérieur, n'ont produit que deux billets. Les blogs sont de manière générale beaucoup moins productifs que les médias professionnels, du fait que leur pratique amateur n'est pas soumise à des contraintes de rentabilité ou de réactivité. Mais une autre raison peut expliquer cet important décalage dans la production des deux catégories. Le 8 mars 2011, le conflit libyen était le sujet le plus traité au sein de notre corpus de sites. Le second sujet le plus traité le même jour était la publication d'un sondage donnant Marine Le Pen, candidate d'extrême-droite, en tête des intentions de vote pour l'élection présidentielle française 
de 2012. C'est ce dernier sujet qui a capté l'attention des blogueurs français, d'abord car la plupart d'entre eux sont engagés dans le débat politique, éventuellement de manière partisane. Une seconde raison est que ce sujet de politique intérieure est pour eux plus aisé à traiter qu'un sujet international tel que le conflit libyen, impliquant des questions diplomatiques et nécessitant une couverture de terrain impossible à réaliser par l'immense majorité des blogueurs. Ici se trouve en quelque sorte un biais d'agenda dans la couverture de l'actualité par les blogs, qui ont tendance à sur-traiter les sujets de politique intérieure et qui manquent de moyens pour couvrir les sujets internationaux. La conséquence, dans nos résultats, est que notre échantillon de blogs a posté ce jour-là 24 billets sur Marine Le Pen, contre seulement 2 sur la Libye. Cela étant, la journée du 8 mars fut également particulièrement intéressante pour ce qui est de la couverture des événements libyens. Le regain de violence ce jour-là dans la ville de Ras Lanouf rendit en effet la question d'une intervention de l'ONU particulièrement cruciale, faisant de la couverture de l'événement, depuis le terrain et dans ses dimensions diplomatiques et géopolitiques, un élément tout à fait central dans la poursuite des événements.

\section{Une analyse lexicométrique du corpus d'articles}

La principale question à laquelle nous nous proposons de répondre est de savoir si les différentes catégories de sites web d'information produisent une grande disparité de cadres d'interprétation sur le conflit libyen, et dans quelle mesure les différentes catégories de sites concourent à cette diversité. Pour répondre à ces questions, nous nous sommes appuyés sur le logiciel Iramuteq ${ }^{1}$, qui repose sur la statistique lexicale (Lebart \& Salem, 1994), et permet de mettre en œuvre une classification hiérarchique descendante du corpus selon la méthode Alceste (Reinert, 2007), très proche de ce que Baumgartner et Mahoney (2008) nomment la « cluster analysis ». Cette méthode opère d'abord un découpage du corpus en unités de contexte (U.C.), qui correspondent à des portions de texte dont la taille est de l'ordre de la phrase. On procède ensuite à un codage de la présence ou de l'absence de chaque

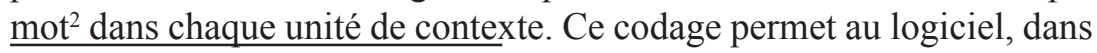

1 Iramuteq est un logiciel libre, sous licence GNU GPL, développé par Pierre Ratinaud, Lerass / Université de Toulouse. http://www.iramuteq.org

2 Par « mot », on désigne ici plus précisément une forme lexicale, c'est-à-dire une graphie, ayant généralement plusieurs occurrences dans le texte. Le mot 
un deuxième temps, de construire des classes de discours à partir des unités de contexte qui contiennent les mêmes mots. Ces classes sont donc constituées par des formes lexicales fortement co-occurrentes dans les unités de contexte, c'est-à-dire des mots très fréquemment utilisés ensemble, formant ce que Reinert (op. cit.) a nommé des « mondes lexicaux $\gg$.

Notre corpus comprend donc 179 articles issus de 41 sites web ayant effectivement publié sur le conflit libyen ce jour-là. Sur le plan lexical, le corpus est constitué de 115,975 occurrences et de 9,890formes. Nous avons ensuite appliqué la classification hiérarchique descendante avec Iramuteq dans le but d'identifier les différents cadres et de mesurer leur usage par les différents sites web de notre échantillon, dans la tradition des travaux de frame mapping (Matthes \& Kohring, 2008) basés sur la définition du cadrage avancée par Entman (1993). La technique du frame mapping automatisée est considérée par Baumgartner et Mahoney (2008, p. 447) comme le moyen d'améliorer la validité et la fiabilité du travail d'identification des cadres: « Methodological advances in computer science now allow much greater use of complex analytic schemes, assisted by computer technologies (not driven by them) to measure the relative use of different frames by different actors in the process ». Les cadres sont ici considérés comme des points de vue, au sens premier du terme, c'est-à-dire des « lieux de la pensée » (Reinert, 2007) à partir desquels se construisent les énoncés. Ces cadres sont ensuite développés dans le discours, en ce qu'ils sont des constructions spécifiques de la réalité concernant un fait ou événement particulier. Cette analyse nous permet donc d'identifier et en quelque sorte d'étiqueter les cadres produits par les sites web d'information, sur la base de cooccurrences de mots statistiquement significatives. Identifier précisément les spécificités lexicales des cadres est une étape essentielle pour les étiqueter, en ce qu'ils sont essentiellement constitués de mots sur-employés, et de mots sous-employés. En effet, Entman (op. cit.) considère que les cadres se définissent aussi bien par ce qu'ils incluent que par ce qu'ils omettent, et que l'omission de définitions ou d'explications potentielles peuvent être tout aussi déterminantes dans le processus d'information. Après avoir identifié les cadres, nous avons donc pu mesurer leur emploi par les différents sites web de notre échantillon, pour tenter de brosser un portrait des principales tendances

« Kadhafi », par exemple, apparait 850 fois dans notre corpus. On parlera alors de la forme «Kadhafi » et de ses 850 occurrences. 
du paysage du web d'information français dans son traitement du conflit libyen.

\section{Résultats et interprétations}

Un panorama des cadres: le dendrogramme

En appliquant la classification hiérarchique descendante, nous avons identifié trois cadres différents dans le traitement du conflit libyen (appelés « classes » dans le schéma ci-dessous) :
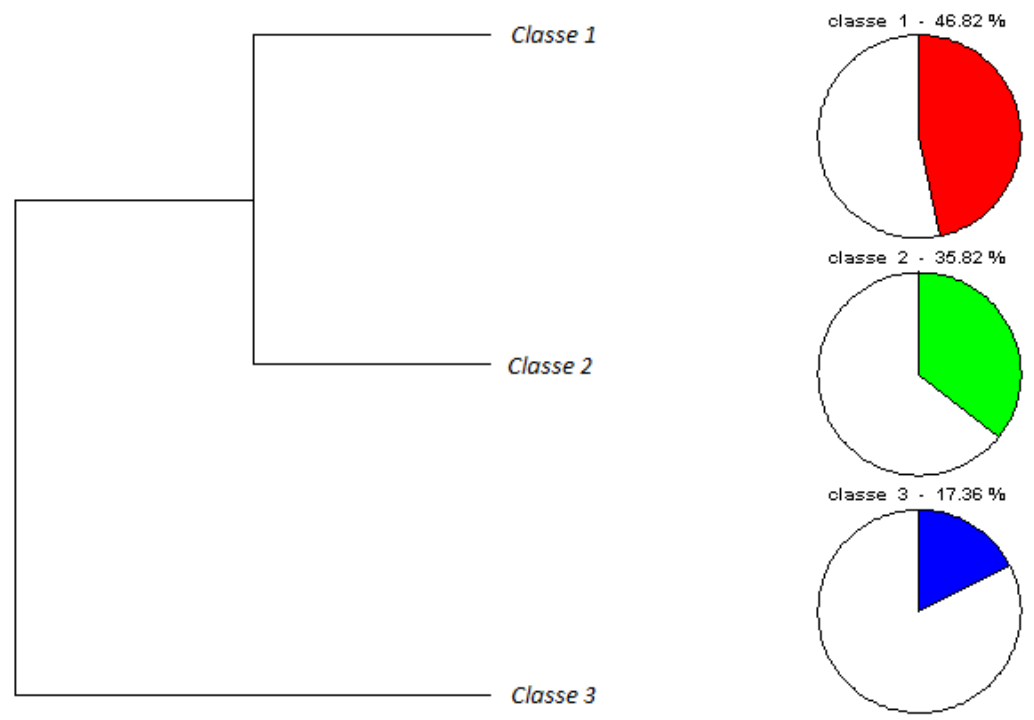

Figure 1. Dendrogramme issu de la classification lexicale du corpus d'articles sur le conflit en Libye

\section{Description détaillée des cadres}

Le premier cadre (correspondant à la classe 1 du dendrogramme) représente environ $47 \%$ du corpus. Il se concentre essentiellement sur les enjeux géopolitiques d'une éventuelle intervention des forces armées internationales en Libye, dans une perspective analytique voire critique, notamment envers les gouvernements français et états-unien. Ce cadrage particulier se manifeste par l'emploi spécifique d'un lexique 
lié aux intérêts géopolitiques et économiques (ex : association des termes « pétrole » - « marché » - « prix », ou mention de «Al Qaïda »), dont les formes les plus significatives peuvent être représentées graphiquement (voir Figure 2) :

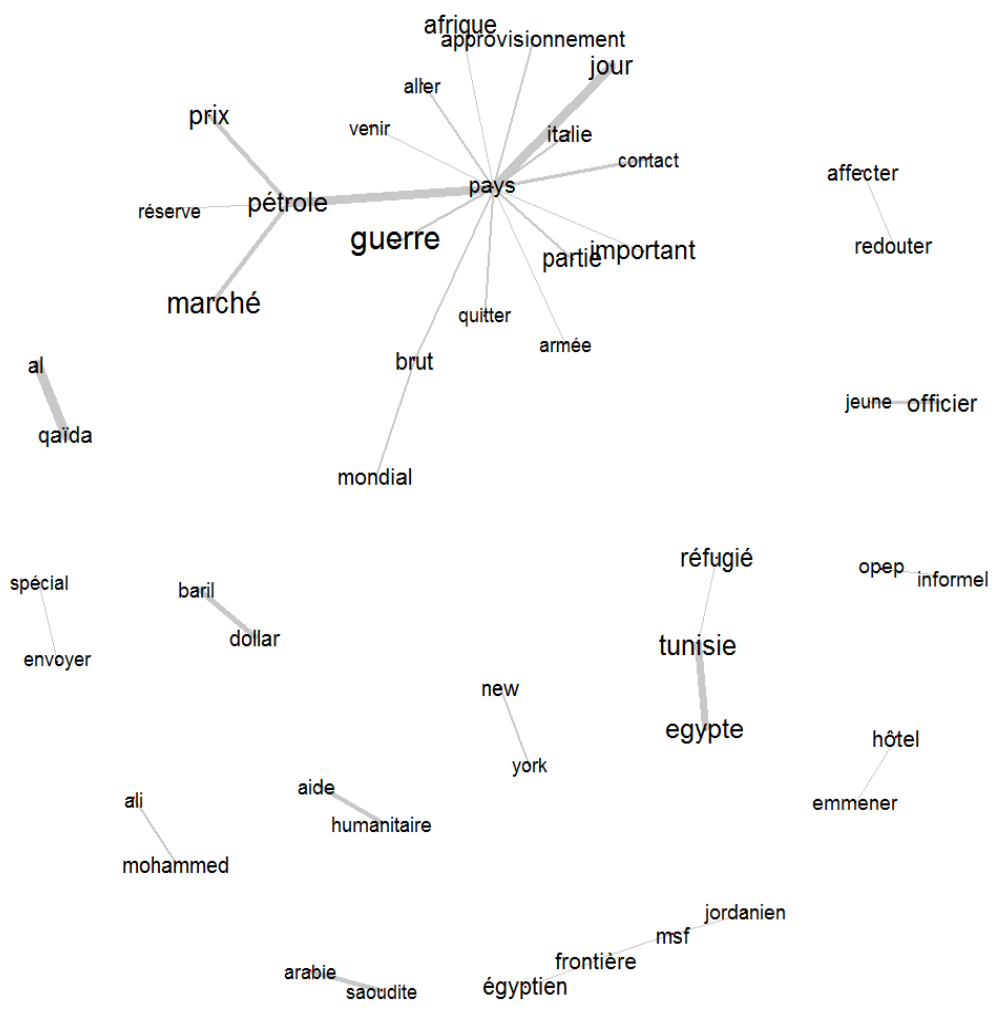

Figure 2. Graphe de cooccurrence des termes les plus significatifs de la classe $1^{1}$

1 Le graphe de cooccurrence est un mode de visualisation proposé par le logiciel Iramuteq. Les termes sont ceux dont les CHI2 respectifs d'appartenance à la classe sont le plus significatif. La taille des mots est proportionnelle à leur fréquence d'occurrence et l'épaisseur des traits dépend également de la force de la relation de cooccurrence entre les différents mots. Pour des questions de lisibilité du graphe, des seuils statistiques ont été définis pour ne garder que les termes les plus significatifs de la classe. 
A titre illustratif, voici un extrait tiré du media natif de l'internet Bellaciao, dont la production est essentiellement réalisée par des non-professionnels, que l'analyse lexicale a identifié comme étant caractéristique de ce cadre :

Par contre, Barack Obama pourrait devoir faire face à un problème plus grave que le Kosovo : l'augmentation des prix du pétrole menace déjà de stopper le rétablissement économique et par conséquent de réduire considérablement ses chances d'être réĺlu l'année prochaine. On ne plaisante pas avec ce genre de choses.

Ce cadre est utilisé par les deux blogs de notre corpus (Sarkofrance et Rebelles.info) et par des sites natifs de l'internet (Alterinfo, Le Post, Agoravox, Le Grand Soir, Bellaciao). Dans une moindre mesure, ce cadre est également véhiculé par le site du quotidien Le Monde. Ces sites web questionnent donc de manière très critique les intentions des pays se proposant de soutenir la révolution libyenne, notamment du point de vue de leur intérêt pour le pétrole libyen et de leur besoin de développer une présence militaire dans le pays.

Le deuxième cadre identifié représente environ $36 \%$ du corpus. Il traite plus spécifiquement de la question des négociations des Nations Unies au sujet d'une intervention militaire et de l'éventuelle mise en place d'une zone d'exclusion aérienne en Libye. Alors que le premier cadre constituait clairement une remise en cause des décideurs politiques internationaux dans leur volonté d'intervenir, ce cadre-ci semble tout à fait neutre, en se focalisant sur un compte-rendu narratif et détaillé des négociations internationales. Ses formes spécifiques sont liées au lexique diplomatique (ex : mention des organisations ONU et OTAN), comme en attestent les formes apparaissant dans le graphe de cooccurrence de la classe 2 (voir Figure 3). 


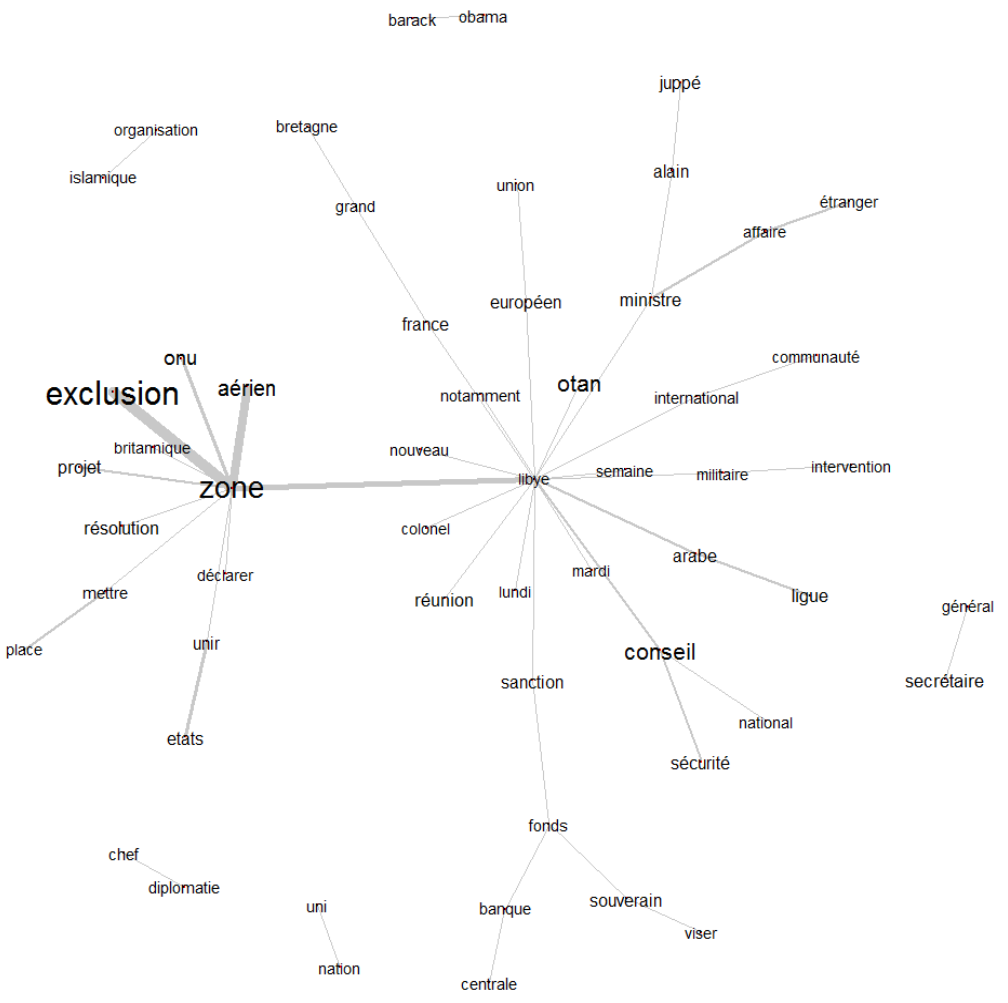

Figure 3. Graphe de cooccurrence des termes les plus significatifs de la classe 2

L'extrait suivant, tiré du site web du quotidien à dominante économique Les Echos, est caractéristique de ce cadre:

La Ligue Arabe a prévu une réunion de crise, samedi, pour évoquer les mesures à prendre pour protéger le peuple libyen, en particulier l'établissement d'une zone d'exclusion aérienne. La France et la Grande-Bretagne préparent un projet de résolution pour le Conseil de sécurité de l'ONU, imposant une zone d'exclusion aérienne au-dessus de la Libye, qu'ils veulent présenter dès cette semaine. 
Ce cadre a été majoritairement utilisé par les médias en ligne, principalement les sites web de quotidiens (Le Figaro, Les Echos, Le Parisien, Aujourd'hui en France, Metro) et d'hebdomadaires (L'Expansion) mais aussi par des sites de radio ou de télévision ( $R M C$, France Info, Europe 1, France 24). Dans une moindre mesure, ce cadre a aussi été employé par certains infomédiaires (MSN Actualités, Orange Actualités) et par des médias natifs de l'internet (Arrêt sur images, Actualités françaises, Planet, Les Infos).

Le troisième et dernier cadre, représentant environ $17 \%$ du corpus, est dédié au reportage de terrain depuis Ras Lanouf, ville dans laquelle une résurgence de la violence était alors observée, comme précédemment mentionnél. Dans ce cadre, le discours est essentiellement factuel, du fait du reportage de terrain, mais des éléments de récit sont également employés, signes d'une volonté de faire vivre et ressentir la situation de guerre au lecteur-internaute. Le lexique spécifique (avec notamment la mention de lieux : « Ras Lanouf », « ville », « ouest ») est lié au vocabulaire militaire et au journalisme embarqué (voir Figure 3).

L'extrait suivant, tiré du site web du média audiovisuel $T F 1$ / LCI, est caractéristique de ce cadre :

L'aviation a bombardé la ville pétrolière de Ras Lanouf, base la plus avancée de l'opposition dans l'Est, faisant un blessé et touchant un immeuble. La banlieue ouest du port pétrolier, à environ $300 \mathrm{~km}$ au sudouest de Benghazi, a été pilonnée et trois personnes ont été blessées.

Ce cadre est nettement sur-employé par les sites web de chaînes de télévision (TF1/LCI, France 2, France 3) dans des articles qui accompagnent très souvent des vidéos tournées sur le terrain et montrant le théâtre d'opérations. Mais il est également utilisé par certains sites de journaux (Libération, Les Echos, Aujourd'hui en France, JDD). Leur production en ligne livre des récits de journalistes ou de témoins embarqués, ou viennent de dépêches d'agences, comme en témoigne notamment la présence de l'AFP dans le lexique spécifique de ce cadre. Quelques infomédiaires (Voilà Actualités, Orange Actualités) et le site natif de l'internet Planet ont également utilisé ce cadre, mais de manière plus ténue.

1 On notera que ce cadre, dont les faits sont géographiquement distincts de ceux des deux autres, est le premier à se distinguer statistiquement par son lexique (voir l'arborescence du dendrogramme en Figure 1). Cette donnée concourt à asseoir la validité et la pertinence de la classification hiérarchique descendante. 


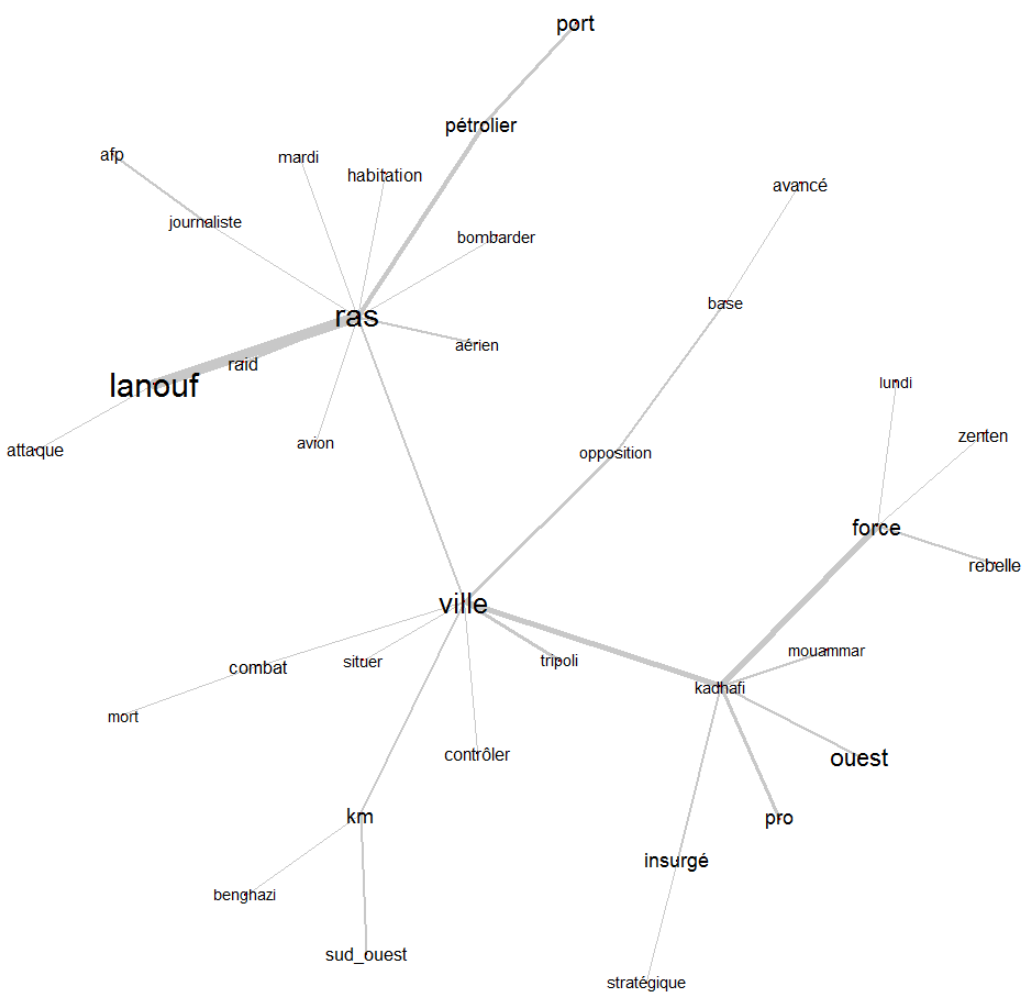

Figure 4. Graphe de cooccurrence des termes les plus significatifs de la classe 3

\section{Enseignements de la recherche et nouvelles perspectives}

Nos résultats viennent confirmer certaines tendances observées dans de précédents travaux, tout en les affinant. Les sites natifs de l'internet et les blogs se distinguent, en se concentrant sur les commentaires et l'alimentation du débat, dans une visée polémique relativement explicite. Si nous avons déjà émis l'hypothèse d'un manque de moyens des blogs pour traiter correctement le sujet (aboutissant à seulement deux billets, comme précédemment mentionné), on peut faire pareille hypothèse pour la plupart des sites natifs de l'internet, dont l'économie fragile ne leur permet pas non plus de couvrir l'événement depuis la 
Libye. On peut alors penser que leur choix de cadre (prioritairement l'analyse critique et polémique) est autant dû aux normes discursives de leur catégorie qu'à l'impossibilité matérielle de produire un autre type de discours. En effet, si l'analyse et l'expression d'opinions constituent indéniablement deux piliers de la politique éditoriale participative de la plupart des sites natifs de l'internet, les faibles sources de financement de ces sites leur imposent la réalisation d'un journalisme principalement «assis », de commentaire et de desk (voir l'article de Smyrnaios dans ce même numéro), moins coûteux que le reportage de terrain caractérisant le journalisme « debout » (Neveu, 2009).

De leur côté, les médias en ligne ont le plus souvent évité l'opinion et le débat au profit de reportages neutralisés et factuels de la situation militaire et diplomatique. Plus précisément encore, chaque média traditionnel (journal, radio, télévision) semble importer sur le web ses propres spécificités discursives: la manière dont chacun couvre le sujet en ligne ne change guère des modalités de traitement qui ont cours chez lui hors ligne.

En effet, les journaux quotidiens et hebdomadaires se sont principalement attachés aux enjeux diplomatiques du débat sur la zone d'exclusion aérienne à l'ONU, là où les sites de télévision et de radio ont privilégié les reportages embarqués en temps réel, incluant des séquences audio ou vidéo prises sur le terrain. En termes de construction du discours, l'objectif des sites web de radios et de chaînes de télévision est de diffuser des nouvelles avec la plus grande réactivité possible, et de proposer à leurs publics de vivre et ressentir les faits en se trouvant au cœur de l'évènement, comme ils le font également hors-ligne. Les déclinaisons web des journaux papier ont plutôt une propension au récit, mais en transmettant aux publics les diverses déclarations et opinions formulées par un large éventail d'acteurs, avec une tendance à privilégier les sources officielles telles que les gouvernements et les organisations internationales. Certains d'entre eux contribuent cependant à alimenter un réel débat sur la situation (par exemple Le Monde), en essayant d'analyser les enjeux de l'intervention internationale. Mais, à regarder de plus près, il s'avère que, tout en posant l'existence d'un débat, les journalistes eux-mêmes ne prennent pas position et évitent d'exprimer des opinions personnelles en faveur d'une position ou une autre. Cette donnée nous remémore l'importance des normes professionnelles dans la presse, au premier rang desquelles figure l'objectivité, qui est sans nul doute une des normes d'écriture les 
mieux respectées dans les grands quotidiens nationaux, en même temps qu'un facteur de crédibilité espérée.

A l'inverse, les sites natifs de l'internet et les blogs ont une identité structurée autour d'autres valeurs. Elles sont étroitement liées à 1'histoire de l'internet, basées sur l'amateurisme et en opposition explicite aux normes journalistiques (Matheson, 2004), quitte à être parfois réappropriées par les professionnels des industries médiatiques traditionnelles (Singer, 2005). Les blogueurs amateurs prennent l'exact contre-pied du traitement journalistique mainstream de l'actualité. Pour ces acteurs de l'information, l'engagement politique et l'expression de l'opinion semblent plus importants que de se fondre dans les normes professionnalistes de la crédibilité journalistique. Toutefois, il existe une distinction importante entre des sites natifs de l'internet revendiquant une telle visée (Agoravox, Alter Info ou Le Grand Soir), et certains d'entre eux qui sont (ou veulent être considérés comme) des médias professionnels (Planet, Les Infos ou Actualités françaises). Là où les premiers sont structurés en associations ou agissent bénévolement, les seconds ont pour objectif de rentabiliser leur production en ligne, essentiellement par la publicité. Cette disparité de fonctionnement socio-économique qui caractérise différentes sortes de médias natifs de l'internet tendrait à jouer un rôle important sur les cadres privilégiés par ces sites.

De manière plus générale, on peut considérer, pour conclure, que nos résultats permettent de mieux comprendre l'arène de l'information en ligne. Ces résultats confirment la complémentarité entre médias professionnels en ligne et sites amateurs, citoyens et/ou et participatifs, que Reese et ses collaborateurs (2007) avaient mis en avant à travers une analyse de la configuration des liens hypertextes entre blogs et sites d'information. En conduisant une analyse de contenu portant sur le paysage de l'information en ligne à l'échelle nationale et englobant l'ensemble des types de sites (médias en ligne et blogs, mais aussi médias natifs de l'internet et infomédiaires), notre étude amène un nouvel élément d'intellection : les sites web ont certes tous tendance à se focaliser sur les mêmes grands sujets de l'agenda médiatique, mais certains d'entre eux adoptent des cadrages radicalement différents pour en traiter. Les sites web amateurs ajoutent clairement une strate d'opinion et de débat (cadrage $\mathrm{n}^{\circ} 1$ dans la présente étude) au traitement journalistique factuel et objectif (cadrages $n^{\circ} 2$ et $n^{\circ} 3$ ) mis en œuvre la plupart du temps par les sites de médias professionnels. 
Pour être encore mieux saisi, ce type de contribution à la sphère publique pourrait par la suite être étudié au moyen d'analyses de discours plus qualitatives. De plus, dans le but de déterminer si à une diversité dans l'offre de contenus correspond une diversité effective dans la réception de l'information par les publics (Van der Wurff, 2011), ce travail pourra être utilement complété par des observations et des enquêtes auprès d'internautes. Enfin, un prolongement encore plus fondamental consistera à voir si ces modalités alternatives de cadrage et de traitement de l'information arrivent à trouver une place, au-delà de l'internet, dans un environnement transmédiatique ${ }^{1}$ de plus en plus interrelié, arène encore plus déterminante pour la visibilité des contenus médiatiques.

\section{Références}

Baumgartner, F.R., \& Mahoney, C. (2008). The Two Faces of Framing: IndividualLevel Framing and Collective Issue Definition in the European Union. European Union Politics, 9(3), 435-449.

Dearing, J.W., \& Rogers, E.M. (1992). Communication Concepts 6. Agenda-Setting, Thousand Oaks, Sage.

Degand, A. (2011). Le multimédia face à l'immédiat. Une interprétation de la reconfiguration des pratiques journalistiques selon trois niveaux, Communication 29(1) | http://communication.revues.org/index2342.html

Deuze, M. (2003). The Web and its Journalisms: Considering the Consequences of Different Types of Newsmedia Online, New Media \& Society 2(5), 203-30.

Domingo, D., \& Paterson, C. (2011). Making Online News, Volume 2, Peter Lang Publishing.

Entman, R. M. (1993). Framing: Toward Clarification of a Fractured Paradigm. Journal of Communication, 43(4), 51-58.

Fenton, N. (2010). New Media, Old News: Journalism \& Democracy in the Digital Age. London: Sage Publications Ltd.

Gamson, W. (1992). Talking Politics. Cambridge University Press.

Gamson, W., \& Modigliani, A. (1989). Media Discourse and Public Opinion on Nuclear

Power: A Constructionist Approach. American Journal of Sociology, 95(1), 1-38.

Gitlin, T. (1980). The Whole World Is Watching. Berkeley, Los Angeles: University of California Press.

1 A ce titre, les enseignements issus de la présente recherche constituent la base du programme OT-Media - Observatoire TransMedia (ANR 2010 CORD 015 06), visant à aller au-delà de l'information en ligne pour l'analyser sur tous les supports (presse, radio, télévision, internet). 
Lebart, L., \& Salem, A. (1994). Statistique textuelle. Paris : Dunod.

Marty, E., Rebillard, F., Smyrnaios, N., \& Touboul, A. (2010). «Variété et distribution des sujets d'actualité sur l'internet. Une analyse quantitative de l'information en ligne », Mots - Les langages du politique, ENS Éditions, 93, 107-126.

Matheson, D. (2004). Weblogs and the Epistemology of the News: Some Trends in Online Journalism, New Media and Society, 6(4), 443-468.

Matthes, J., \& Kohring, M. (2008). The Content Analysis of Media Frames: Toward Improving Reliability and Validity. Journal of Communication, 58(2), 258-279.

Moirand, S. (2007). Les discours de la presse quotidienne. Observer, analyser, comprendre. Paris : Presses Universitaires de France.

Mosco, V. (2009). Review Essay: Approaching Digital Democracy. New Media \& Society, Published online before print November 24.

Neveu E. (2009), Sociologie du journalisme, Paris : La Découverte

Peters, J. (2004). "The Market-Place of Ideas": A History of a Concept. In Calabrese, A., \& Sparks, C., (Eds) Toward a Political Economy of Culture: Capitalism and Communication in the Twenty-first Century, Lanham, MD: Rowman and Littlefield, pp. $65-82$.

Rebillard, F. (2006). Du traitement de l'information à son retraitement. La publication de l'information journalistique sur l'internet, Réseaux, 137, 29-68.

Rebillard, F., \& Smyrnaios, N. (2010). Les infomédiaires au cœur de la filière de l'information en ligne. Les cas de Google, Wikio et Paperblog, Réseaux 160161/2010, 163-194.

Reese, S., Rutigliano, L., Hyun, K., \& Jeong, J. (2007). Mapping the Blogosphere. Professional and Citizen-based Media in the Global News Arena, Journalism,8(3), 235-261.

Reinert, M. (2007). Contenu des discours et approche statistique. In Gauzente, C., \& Peyrat-Guillard, D. (éds.). Analyse statistique de données textuelles en sciences de gestion. Cormelles-le-Royal : EMS, p. 21-45.

Singer, J. B. (2005). The Political J-Blogger: 'Normalizing' a New Media Form to Fit Old Norms and Practices, Journalism, 6(2), 173-198.

Smyrnaios, N., Marty, E., \& Rebillard, F. (2010). Does the Long Tail Apply to Online News? A Quantitative Study of French-speaking News Websites. New Media and Society 12(8), 1244-1261.

Smyrnaios, N. (2012). "How Does News Infomediation Operate Online? The Examples of Google and Facebook", presentation in the 10th World Media Economics \& Management Conference, Thessaloniki, May 23-26.

Van der Wurff, R. (2011). Do Audiences Receive Diverse Ideas from News Media? Exposure to a Variety of News Media and Personal Characteristics as Determinants of Diversity as Received, European Journal of Communication, 26(4), 328-342 